\title{
Estimating the Number of Factors in Exploratory Factor Analysis via out-of-sample Prediction Errors
}

\author{
Jonas Haslbeck ${ }^{1}$ and Riet van Bork ${ }^{1,2}$ \\ ${ }^{1}$ Psychological Methods Group, University of Amsterdam \\ ${ }^{2}$ Center for Philosophy of Science, University of Pittsburgh
}

\begin{abstract}
Exploratory Factor Analysis (EFA) is one of the most popular statistical models in psychological science. A key problem in EFA is to estimate the number of factors. In this paper, we present a new method for estimating the number of factors based on minimizing the out-ofsample prediction error of candidate factor models. We show in an extensive simulation study that our method slightly outperforms existing methods, including parallel analysis, AIC, BIC, RMSEA, and Exploratory Graph Analysis. In addition, we show that, among the best performing methods, our method is the one that is most robust across different specifications of the true factor model. We provide an implementation of our method in the R-package fspe.
\end{abstract}

\section{Introduction}

Exploratory factor analysis (EFA) is one of the most used statistical models in psychology. It is central to the validation of measurement scales (Campbell-Sills \& Stein, 2007; Diemer, Rapa, Park, \& Perry, 2017), identifying latent dimensions (Carragher et al., 2016), and summarizing data (Watson, 2017). The key problem in an EFA is to select the number of factors, which is a nontrivial problem, because adding more factors will always improve the fit to the data, even if the true number of factors is small.

Most of the existing methods to select the number of factors fall into two categories. The methods in the first category analyze the pattern of eigenvalues of the covariance matrix to determine the number of factors. A popular example is the scree-plot, which plots the eigenvalues in decreasing order. One looks for an "elbow" in the plotted curve and selects the number of factors to be equal to the number of eigenvalues before the "elbow" (Cattell, 1966). A less subjective method based on eigenvalues is the Kaiser-Guttman criterion, which estimates the number of factors as the number of eigenvalues above 1 (Guttman, 1954; Kaiser, 1960). However, the Kaiser-Guttman criterion ignores sampling variability and is therefore prone to overestimating the number of factors (Fabrigar, Wegener, MacCallum, \& Strahan, 1999). This motivated parallel analysis, which generates data from diagonal covariance matrices, orders the empirical and random eigenvalues from large to small, and selects the number of factors as the largest value $j$ such that the first $j$ eigenvalues for the actual data exceed the mean of those in the random data sets (Horn, 1965). This method has been further improved by using a high quantile instead of the mean as a threshold (Glorfeld, 1995; Harshman \& Reddon, 1983; Weng \& Cheng, 2005).

The second category approaches the estimation of the number of factors as a model-selection problem (Preacher, Zhang, Kim, \& Mels, 2013). Since the factor model with the highest number of factors always fits best, estimating the number of factors in the population requires a trade-off between model fit and complexity. A popular choice is the Bayesian Information Criterion (BIC; Schwarz, 1978) which in fact has been first introduced to estimate the number of factors in EFA as an improvement over the Akaike Information Criterion (AIC; Akaike, 1973). An alternative is the Root Mean Squared Error of Approximation (RMSEA), which can be regarded as an estimate of model misfit per degree of freedom (Browne \& Cudeck, 1992). Yet another method of this type is the Minimum Average Partial (MAP) test, which looks for the average residual partial correlations

Corresponding author: jonashaslbeck@gmail.com | www.jonashaslbeck.com 
for different numbers of factors, and selects the one with the lowest value (Velicer, 1976). The MAP test is strictly speaking not a test, but it implicitly involves the sequential search for the most parsimonious model (Ruscio \& Roche, 2012). Next to those two main categories, there are a number of additional methods such as the Very Simple Structure (VSS) method (Revelle \& Rocklin, 1979) which tries to recover simple structures that are easy to interpret, or the recently proposed Exploratory Graph Analysis (EGA) (Golino \& Demetriou, 2017; Golino \& Epskamp, 2017), which first estimates a regularized partial correlation matrix and subsequently applies a community detection algorithm to determine the number of factors.

The contribution of this paper is to introduce a new method to estimate the number of factors in EFA that is based on minimizing the out-of-sample prediction across EFA models with different numbers of factors. We demonstrate in a simulation study that our method slightly outperforms existing methods and is particularly robust across scenarios. Finally, we provide the R-package fspe which implements our new method.

\section{New Method based on out-of-sample Prediction Errors}

Our new method falls in the second category of methods, that is, methods that approach the estimation of the number of factors in EFA as a model selection problem. From this perspective, the goal is to compare the models with $1,2, \ldots, p$ factors, where $p$ is the number of variables, and select the model that has the lowest expected prediction error in the population. This is a nontrivial task because the model that minimizes prediction error in the sample typically does not minimize the prediction error in the population. Selecting the number of factors based on the prediction error within the considered sample often leads to overfitting, that is, selecting a too complex model that fits the current sample well, but doesn't generalize well to new samples (Hastie, Tibshirani, \& Friedman, 2001). In the context of EFA, the model with $p$ factors will always have the lowest prediction error within sample, but might have a very high error in new samples. Some of the selection criteria mentioned above, such as the BIC, deal with overfitting by penalizing the number of free parameters in the model relative to the model fit.

In this article, we use a more data-driven approach by using out-of-sample prediction errors to approximate the prediction error in the population. This works because the population can be seen as an infinitely large sample of new (i.e., out-of-sample) data and therefore the expected prediction error in the population is equal to the prediction error in an infinitely large sample of new data. This prediction error is often referred to as out-of-sample prediction error, to emphasize that the prediction error is computed on new data that are independent of the data used to estimate the model. Of course, we do not have an infinitely large sample, but we can estimate this theoretical prediction error in a finite sample. This idea is used widely in machine learning, where model selection is performed by evaluating the models' relative performance in a test dataset that has not been used for parameter estimation (e.g., Bishop, 2006). For the problem studied in this paper, this means that we fit factor models with $1,2, \ldots, p$ factors on one part of the data (often called training dataset) and we evaluate the prediction error of those models in the (out-of-sample) test dataset. Finally, we select the model whose number of factors minimize the out-of-sample error in the test dataset.

A drawback of splitting the data into training/test sets is that we do not use all the data for estimation, because we need to keep some of the data in the test set to compute the out-of-sample prediction error. A way to address this problem is $\mathrm{k}$-fold cross-validation, which creates $k$ train-test splits in the data, and returns a prediction error averaged across the $k$ test datasets (e.g., Hastie et al., 2001). Although this method introduces bias in the estimate of the out-of-sample prediction error, this bias is usually justified by the efficiency that we gain from using all data for estimation within the cross-validation scheme. We therefore adopt a cross-validation scheme to estimate the out-of-sample prediction error in our method. A possible advantage of the method we present here is that it provides a relatively direct estimate of the quantity we want to minimize theoretically, and does not rely on distributional assumptions or asymptotic theory.

A crucial ingredient of our proposed method is that the factor model can be used to compute prediction errors. At first sight, a factor model cannot be used to make predictions about variables, because the variables that should be predicted are themselves required to estimate the factor scores, so that the factor scores cannot be used to predict these variables. We therefore turn to the model-implied covariance matrix $\Sigma$ and its inverse $K=\Sigma^{-1}$. Specifically, we make use of the relation between the entries of the inverse covariance matrix, partial correlations, and standardized 
regression weights

$$
\frac{\beta_{i, j}+\beta_{j, i}}{2}=\operatorname{cor}\left(X_{i}, X_{j} \mid X_{-(i, j)}\right)=-\frac{K_{i, j}}{\sqrt{K_{i, i}} \sqrt{K_{j, j}}},
$$

where $X_{i}$ denotes column $i$ in the data $X \in \mathbb{R}^{n \times p}$. The index $-(i, j)$ denotes the set of all variables except $i$ and $j, \beta_{i, j}$ is the standardized coefficient for variable $X_{j}$ in the regression of all variables on $X_{i}$, and $K_{i, j}$ is the entry at row $i$ column $j$ in matrix $K$ (see also Epskamp, Waldorp, Mõttus, \& Borsboom, 2018; Lauritzen, 1996). This means that we can use the implied covariance matrix to define $p$ regression equations, in which $X_{i}$ is predicted by all other variables $X_{-i}$, which allows us to compute a prediction error for each variable $X_{i}$. In order to make predictions with standardized regression weights, the data needs to be standardized. However, this standardization does not impact the model selection procedure because factor models are invariant to linear transformations.

Browne and Cudeck (1989) suggested a similar method which divides the data in two parts. In the first part of the data they fit candidate factor models and compute their respective implied covariance matrices. In the second part of the data they compute the sample covariance matrix. Then, they select the factor model which minimizes the distance between the implied covariance matrix and the sample covariance matrix. That is, they perform cross-validation with two folds. However, their method is different from ours because they approximate an out-of-sample estimation error based on covariance matrices, and not based on predictions of the variables themselves. To our knowledge, the performance of their method has not yet been systematically evaluated and we therefore include it in the simulation study below. Browne and Cudeck $(1989,1992)$ also developed an improved version of their method that no longer splits the data in halves but uses all of the data. This expected cross-validation index (ECVI) is linearly related to the AIC, when maximum likelihood estimation is used (Browne \& Cudeck, 1992). Since ECVI and AIC give the same rank order of models, we include the AIC in our simulation study.

To summarize our method, we fit factor models with $1,2, \ldots, p$ factors, and for each model we compute the model-implied covariance matrix, which, for each factor model, allows us to obtain $p$ regression models. We use these models to compute for each variable $X_{i}$ an approximation of the out-of-sample prediction error using cross-validation. We then average the prediction error across folds and variables to obtain a single aggregate prediction error for each considered factor model. Finally, we select the model with the number of factors that minimizes this prediction error. We make an implementation of our method available in the R-package $f$ spe which is available on the Comprehensive R Archive Network (CRAN) and in Appendix A, we provide a brief example of how to use this implementation to select the number of factors in an empirical dataset.

\section{Determining Performance via Simulation}

The goal of this simulation is to determine the performance of our method compared to the existing methods discussed above in scenarios that are typical to psychological research.

\subsection{Considered Scenarios \& Data Generation}

We generate continuous data from factor models that vary in the following characteristics: (a) the number of factors $(1,2,4$, or 6$)$; (b) the number of variables that load on each factor $(3,6$, or 12); and (c) the correlation between each factor pair (no correlation (0), medium correlation $(0.40)$, high correlation $(0.80))$. The correlations are chosen to cover most of the correlations likely encountered in empirical data. The factor loadings on a given factor typically vary in empirical data. Accordingly, to create realistic simulation scenarios, we draw loadings from a uniform distribution $\mathcal{U}(0.3,1)$ and we choose a diagonal residual covariance matrix with variances set to 1 . We did not include cross-loadings, so that each item loaded on only one factor. This setup results in correlations between variables within factors which we deem realistic for psychological datasets. For example, in the scenario with six variables per factor and zero correlations between factors, the mean correlation between variables is equal to $0.37(S D=0.29)$. In addition, we varied the sample size in 12 steps from 100 to 5000 on a logarithmic scale. We chose these values to cover the range of typical datasets in psychological research and used a log-scale to focus on the smaller, more common, datasets.

This design results in $4 \times 3 \times 3 \times 12=432$ scenarios and is therefore the largest fully crossed simulation study on estimating factors in EFA we are aware of (see Appendix F for a summary of 
the design and results of these simulation studies; Crawford et al., 2010; Golino \& Demetriou, 2017; Golino \& Epskamp, 2017; Green, Levy, Thompson, Lu, \& Lo, 2012; Keith, Caemmerer, \& Reynolds, 2016; Ruscio \& Roche, 2012; Velicer, Eaton, \& Fava, 2000; Zwick \& Velicer, 1982). However, perhaps the most important way in which our simulation study extends existing simulations is that we introduce variation in factor loadings, which are typically treated as being all equal. Varying the factor loadings introduces a considerable amount of sampling variance, which is why we repeat our design 200 times. We generated the data with the R-package lavaan (Rosseel, 2012) version 0.6-5. The code to fully reproduce our simulation study and all results reported in this paper can be found at https://github.com/jmbh/EstimateFactorsEFA.

\subsection{Estimating the Number of Factors}

Many methods exist for estimating the number of factors in EFA and including all of them in our simulation study would not be feasible. Therefore, to evaluate the usefulness of our new method, we only compare it to the currently best-performing methods. To this end, we reviewed past simulation studies and included those methods that performed best in those simulation studies (see Appendix F). Based on this review, we consider parallel analysis, EGA, AIC, BIC, MAP, Kaiser-Guttman criterion, VSS, the RMSEA, the method using estimation errors on the covariance matrix (CovE), and our new method based on prediction errors (PE).

In all methods that involve fitting factor models, we use the implementation of EFA in the psych package (Revelle, 2021b) version 2.0.9, with the default oblimin rotation and using Maximum Likelihood estimation. We compared the candidate factors $1,2, \ldots, 10$. However, in some cells we considered fewer factors in order to ensure that all candidate models are identified. In the scenarios with three variables per factor and one factor, all factor models would result in zero or negative degrees of freedom, and we therefore excluded this scenario from our simulation. For MAP, VSS, Kaiser-Guttman, RMSEA and parallel analysis we use the implementations in the vss() function in the psych package, using rotate = "oblimin", which is the default in the $\mathrm{fa}()$ function (the function for estimating an EFA in psych). The MAP algorithm implemented in the psych package is that described in Velicer (1976). The VSS procedure implemented in the psych package is that described in Revelle and Rocklin (1979). In the VSS procedure, we used complexity 1, which means that only one loading per variable (the largest) is not set to 0 . The fit of this simple structure is compared for each model with $1,2, \ldots, p$ factors, selecting the best fitting model. For the Kaiser-Guttman criterion we selected the number of factors for which the eigenvalues $>1$.

We used two procedures based on the RMSEA. In one procedure, we selected the smallest number of factors for which the RMSEA drops below 0.05. In a second procedure we calculated a $95 \%$ confidence interval around the RMSEA, and selected the smallest number of factors for which the lower bound is smaller than 0.05 (RMSEA.LB; Preacher et al., 2013). Both of these procedures are based on a cut-off value of RMSEA $<0.05$ for close fit (Browne \& Cudeck, 1992). Browne and Cudeck (1992) base this cut-off on a "substantial amount of experience" with RMSEA measures, and note that the subjective nature of this cut-off can be seen as similarly subjective to setting a significance level of 0.05 . While the use of an arbitrary cut-off might not be optimal, we rely on automated versions of these measures to be able to implement them in the simulation study.

In parallel analysis, the empirical eigenvalues have to be above the $95 \%$ quantile of the corresponding eigenvalue based on random data. We specified $f a=~ " f a "$ in the fa.parallel() function to use the eigenvalues of factor analysis instead of those of the principal components. For the EGA method we use the EGAnet package (Golino \& Christensen, 2020) version 0.9.8. The EGA method is based on estimating regularized partial correlations using the glasso algorithm (Friedman, Hastie, \& Tibshirani, 2008) and performs model selection using the Extended Bayesian Information Criterion (EBIC; Foygel \& Drton, 2010). The EBIC has a tuning parameter which we set to $\gamma=0.50$, which is the default setting in the EGAnet package. In addition, we compared the $1,2, \ldots, p$ candidate factors based on the AIC $=\chi^{2}-2 d f$ and BIC $=\chi^{2}-d f * \ln (N)$, and selected the number of factors that minimized AIC/BIC. ${ }^{1}$

Some of the considered methods fit better with the logic of principal components analysis (PCA) than factor analysis (e.g., MAP and the Kaiser-Guttman criterion; Kaiser, 1991; Velicer, 1976). Since we are concerned with the dimensionality problem in the context of EFA, such methods might

\footnotetext{
${ }^{1}$ This version of the BIC is used in the psych package. Note that $\chi^{2}-2 d f$ renders the same results as $\chi^{2}+2 k$, and $\chi^{2}+d f * \ln (N)$ renders the same results as $\chi^{2}+d f * \ln (N)$, where $k$ is the number of free parameters, since in both cases the formulas differ only by a constant.
} 
have a disadvantage compared to methods that are specifically developed for the factor analysis context (e.g., model-selection measures such as RMSEA, AIC and BIC). It is therefore important to interpret the results of this simulation study only for the EFA context; in the context of PCA, other methods might be preferable. We nonetheless include measures that originate from PCA in this simulation study because it is possible that they outperform other measures also in the context of EFA. For example, Velicer (1976), who developed MAP, notes that MAP, which is developed in the context of component analysis, is appropriate as a first-stage solution for factor analysis. For measures for which there exists both a version for EFA and for PCA (e.g., parallel analysis), we use the EFA version.

For our method based on out-of-sample prediction error we use the package $f$ spe version 0.1.0 (Haslbeck \& van Bork, 2021). This implementation uses the psych package to perform EFA with oblimin rotation. This package also implements a more flexible version of the method proposed by Cudeck and Browne (1983). We use 10 folds in the cross-validation scheme, which has been shown to result in acceptable bias and variance (Kohavi, 1995). We also investigated performance as a function of the number of folds in the present context and found that 10 folds performed best (for details see Appendix B). A common strategy to increase the performance of model selection with cross-validation is to re-run the cross-validation scheme repeatedly and aggregate the results. We found that re-running the cross-validation scheme 10 times and aggregating the estimates with a majority rule led to a very small improvement in performance (for details see Appendix C). We therefore use the version with 10 re-runs for the out-of-sample prediction methods in this paper.

In our simulation study we compare methods in how well they identify the number of factors of the data-generating model (i.e., the true model). However, identifying properties of the true model is not necessarily the only goal one can have when selecting a model. This is especially relevant if it is unrealistic that the true model is within the considered model class. Some of the methods for selecting the number of factors we consider in this study are optimized for the goal of selecting the model with the best generalizability (i.e., the ability to generalize well to new data from the same underlying process Preacher et al., 2013). The AIC, for example, is designed for the goal of generalizability and is equivalent to the Expected Cross Validation Index (ECVI Cudeck \& Browne, 1983) when using Maximum Likelihood estimation. The PE method we propose also selects a model based on minimizing out-of-sample (i.e. generalization) error. Although these methods optimize generalizability, it is likely that these also do well in selecting the true model, if the true model is within the considered model class, because the predictions of the true model tend to generalize well to new samples. For example, the AIC, while designed for generalizability, is shown to perform well in recovering the true number of factors, although only for small N (Akaike, 1998; Preacher et al., 2013). For our purposes here it is important to note that we compare the methods based on whether they can recover the true number of factors in sample sizes that are typically encountered in applied research, which means that the results should also be interpreted in light of that goal.

Note that the methods included are all automated procedures that select the number of factors on purely statistical grounds and do not make use of the expertise of the researcher to select the number of factors.

\subsection{Results}

The proportion of correct classifications (or accuracy) averaged across all conditions and with 200 iterations was highest for our out-of-sample prediction error method (0.813), closely followed by parallel analysis (0.798), EGA (0.791), CovE (0.788) and the AIC (0.753). Since evaluating the variability in performance in different methods is more meaningful when keeping the sample size $N$ fixed, Figure 1 displays the accuracy of each method averaged across $4 \times 3 \times 3=36$ conditions and the 200 iterations for $N=100$ (light grey), $N=847$ (dark grey) and $N=5000$ (black). The methods are ordered decreasingly based on the average accuracy across all conditions in the entire simulation design ${ }^{2}$. The vertical bars represent the $10 \%$ and $90 \%$ quantiles of the accuracy distribution across conditions (for fixed $N$ ) and therefore quantify how much the performance varies across different specifications of the data generating factor model. EGA returns no estimate of the number of factors if an insufficient number of partial correlations are estimated nonzero ( $1.03 \%$ of cases in our simulation). We coded such cases as incorrectly classified.

\footnotetext{
${ }^{2}$ To check how stable the ordering is, we divided the 200 iterations in 20 equally sized batches. The ordering of the methods was the same in $18 / 20$ batches, indicating high stability of the ordering.
} 


\section{Figure 1}

Mean and $10 \%$ and $90 \%$ quantiles of the accuracy for the considered methods across conditions for fixed sample sizes $N=100,847$ and 5000. $P E=$ Prediction Error based method, Parallel = parallel analysis, EGA = Exploratory Graph Analysis, CovE = error computed on out-of-sample covariance matrix, BIC = Bayesian Information Criterion, RMSEA = Root Mean Squared Error of Approximation, MAP = Minimum Average Partial test, $K-G=$ Kaiser-Guttman criterion, VSS = Very Simple Structure. The horizontal ordering of methods is decreasing based on the accuracy across all conditions, including all $N$-variations.

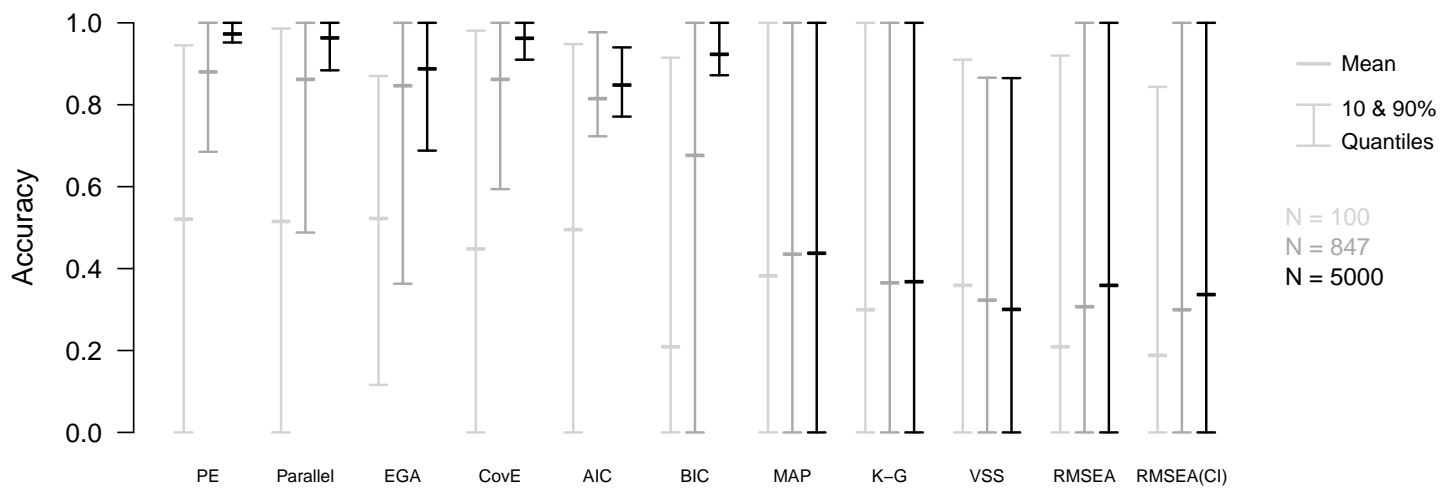

We see that for all methods the performance increases with increasing sample size $N$, as expected. The only exception is the VSS approach, in which increasing sample sizes lead to lower performance. The mean performance of the top four methods (PE, Parallel, EGA, and CovE) is very similar within each of the three displayed sample size variations. However, the methods differ clearly in how much their performance varies across conditions: accuracy varies the least for PE and CovE, followed by parallel analysis and EGA.

We now turn to the performance as a function of the different simulation scenarios. Figure 2 displays the performance of the five best performing methods (PE, parallel analysis, EGA, CovE, and AIC) as a function of the number of true factors, the number of variables per factor, the correlations between factors and the sample size. For all five methods it generally holds that performance decreases for (a) smaller sample sizes, (b) more factors, (c) higher correlations between factors and (d) fewer variables per factor. However, the five methods are differently affected by those five characteristics. The AIC is performing well for small $N$ but its performance does not seem to converge to 1 when $N$ is large, like for the other methods. Higher correlations between factors drastically reduced the performance of EGA in the conditions with three variables per factor. Few variables per factor mostly decreased the performance of parallel analysis in conditions with a small number of factors, and decreased the performance of CovE mostly in conditions with a large number of factors.

The PE and CovE methods perform similar for few variables per factor, but PE outperforms CovE in conditions with more variables per factor and with correlations between factors. This explains the overall higher performance of PE (see Figure 1). It is interesting that PE outperforms CovE in these conditions, since the PE method makes predictions based on the model-implied covariance matrix that is also used by the CovE method. PE and parallel analysis perform similarly, except that parallel analysis performs better with few variables per factor, and PE performs better with more variables per factor. Overall, the PE method is among the best performing methods in all conditions and its performance converges to 1 as a function of $N$. This explains its relatively low variability in performance across simulation conditions displayed in Figure 1.

So far, we only considered the accuracy of methods, but did not investigate whether errors are due to over- or under-estimation of the number of factors. In Figure 3, we display the bias for each method in each condition. The bias is calculated as the estimated number of factors minus the true number of factors. Thus, values below 0 indicate underestimation of the number of factors. These results show that bias is always due to under-factoring, with the exception of EGA in scenarios with many variables and high correlations between factors. 


\section{Figure 2}

The accuracy of our method based on out-of-sample prediction errors (PE), parallel analysis, Exploratory Graph Analysis (EGA), the squared error based on the out-of-sample covariance matrix (CovE), and $A I C$ as a function of the number of true factors, the number of variables per factor, the correlation between factors and the sample size. Note that for the cell with 3 variables per factor a factor model with 1 factor is not identified and was therefore not included in the simulation. In addition, for the cells with 1 factor there are no correlations between factors, and we therefore do not vary this condition for models with 1 factor.

Factors $=1$
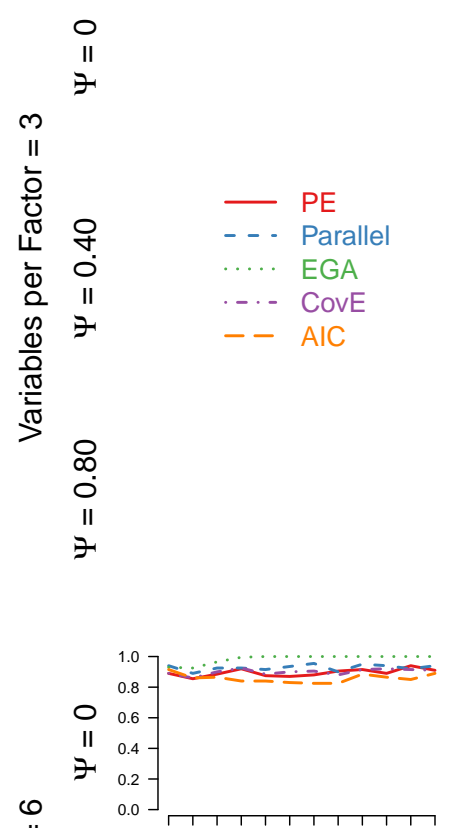

인.

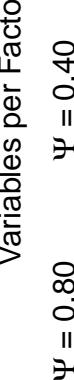

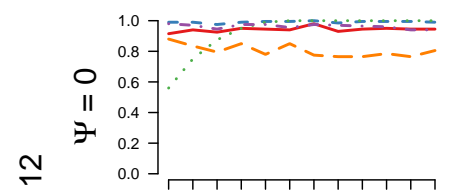

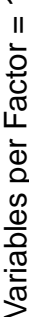

$\infty$

$\infty$
0
$\vdots$
II
Factors $=2$
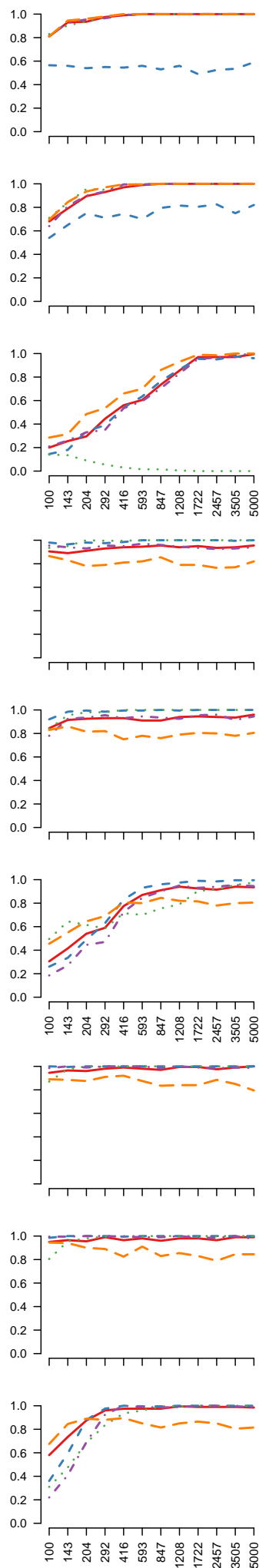

Factors $=4$
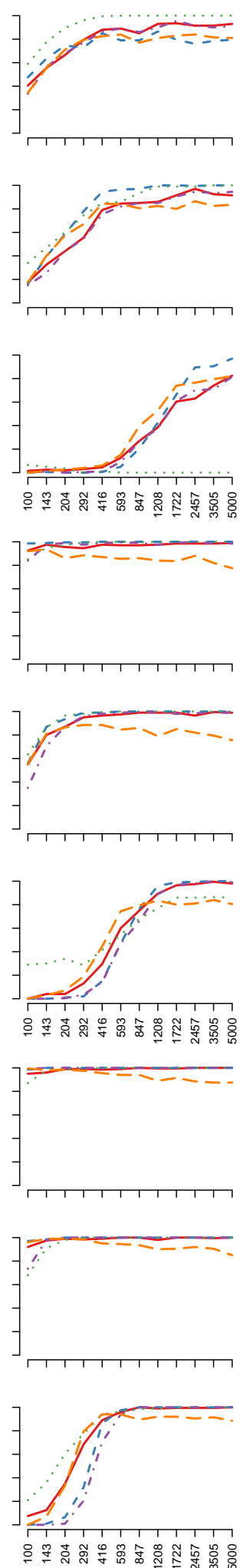

Factors $=6$
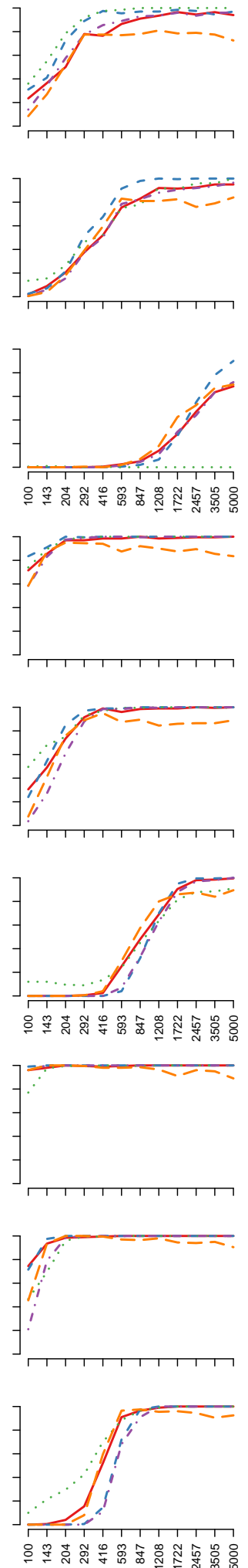


\section{Figure 3}

The bias of all methods considered in Figure 2. The bias is calculated as bias = estimated - true. That is, negative values indicate underestimation of the true number of factors. We see that all bias is due to under-factoring.

Factors $=1$
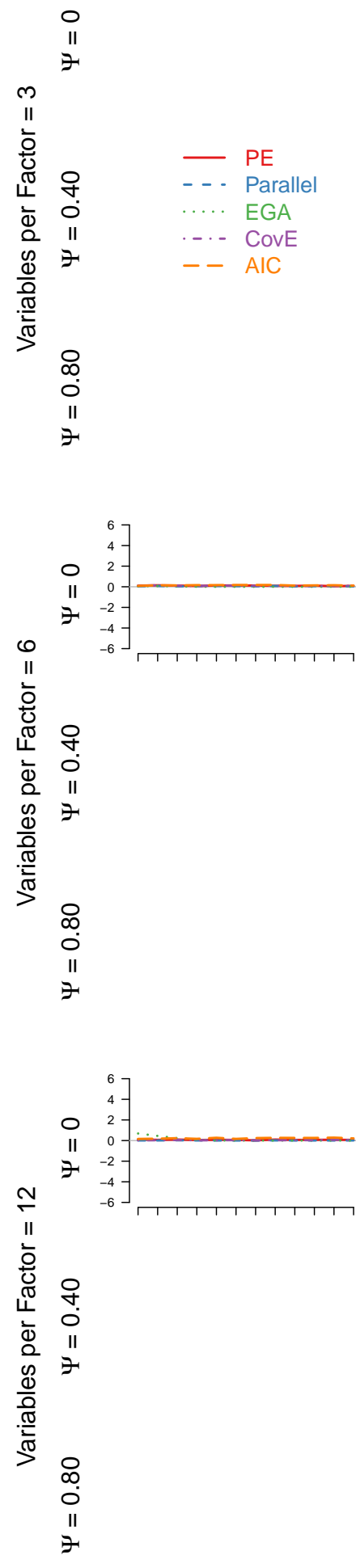

Factors $=2$
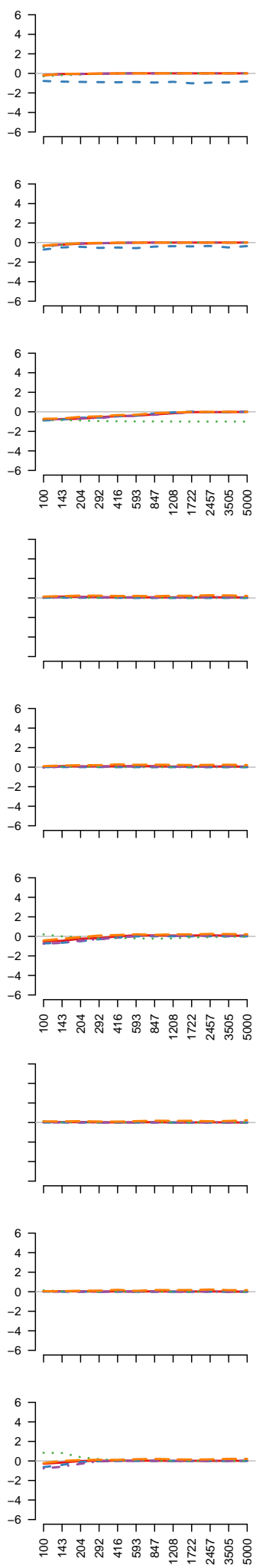

Factors $=4$
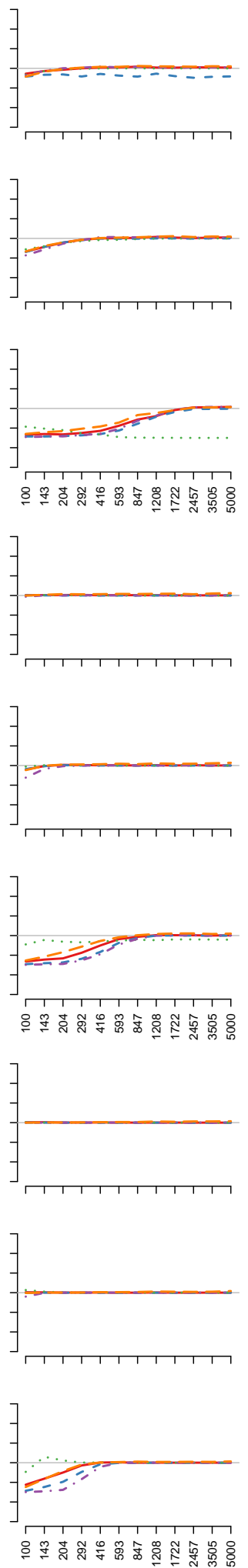

Factors $=6$
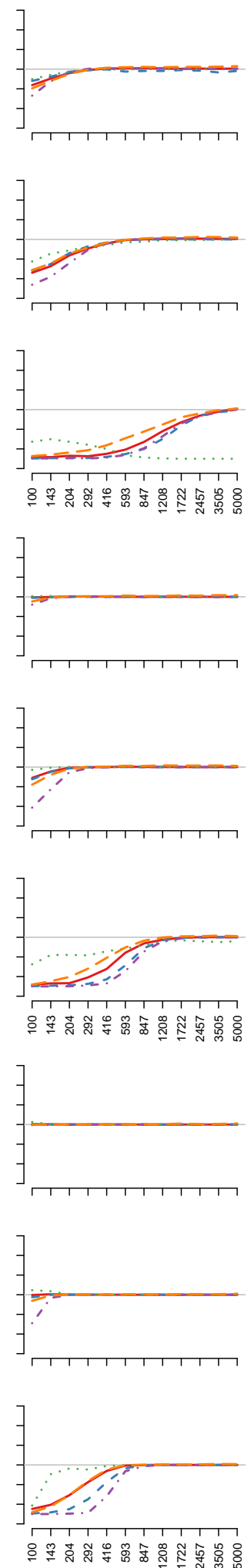


\section{Discussion}

We proposed a new method to select the number of factors in Exploratory Factor Analysis using out-of-sample prediction errors, and demonstrated its performance relative to many other popular methods in an extensive simulation study. We found that our method on average slightly outperforms the best-performing existing methods and is also more robust across the many different specifications of true factor models that we considered.

A few simulation results stood out. We found that parallel analysis performs poorly in the scenario of two true factors, three variables per factor and no (0) or medium (0.4) correlations between factors. Specifically, parallel analysis incorrectly selects 0 factors in those situations. The reason for this perhaps counter-intuitive result is that the $95 \%$ quantile of the largest eigenvalue in the simulated data is very large compared to the remaining eigenvalues, and the largest empirical eigenvalue does not tend to reach this high threshold if the number of variables per factor is small. This leads to a high threshold for the empirical eigenvalue to exceed, thereby leading to an incorrect estimate of 0 factors in these situations. Interestingly, this problem persists even when increasing the sample size considerably. We provide a more detailed discussion of this phenomenon and additional analyses of the performance of parallel analysis in Appendix D.

Another interesting finding is that the CovE method performs differently than the AIC. This is surprising because Browne and Cudeck (1989) introduced the expected cross-validation index (ECVI) as a theoretical approximation of the error used in the CovE method and state that the ECVI selects the same model as the AIC. Our results show that the AIC, and thereby the ECVI suggested by Browne and Cudeck (1989), poorly approximates the out-of-sample error on the covariance matrix. However, an in-depth analysis of the nature of the discrepancy between the ECVI/AIC and the CovE method in these cases is beyond the scope of this paper.

Results of a recent simulation study of Golino et al. (2020) indicated that EGA performed somewhat better than parallel analysis across the total set of conditions, but, in line with our results, EGA and parallel analysis both showed a high accuracy (accuracies of resp. $88 \%$ and $83 \%$ across conditions) and outperformed other criteria included. In line with our results, high correlations (0.7) between factors in combination with small numbers of variables (3) per factor negatively impacted the performance of EGA more than of parallel analysis, and, also in line with our results, their results show that parallel analysis has a negative bias (underestimation of number of factors) in a large part of the conditions, while EGA has not.

Overall, we found that different methods are better in different situations. For example, parallel analysis is performing worse than the PE method for true factor models with two factors, but tends to outperform the PE method if there are four or six factors in combination with a high sample size. These kind of results can be used by researchers who have some expectations regarding the true model, either based on theory or existing data analyses. If no such knowledge is available, the PE method proposed here is a good option due to its high performance and robustness.

An additional way to determine the number of factors is to combine the estimates from different methods. For example, one could use a majority rule across the best performing methods. In Appendix G, we show that a majority rule of the Top 3 methods outperforms all other (composite) methods by a small margin. This is possible because it happens to be the case that in each scenario only one of the three methods shows relatively low performance. However, it is unclear how well these results generalize beyond the scenarios studied in our simulation study and we leave a thorough investigation of such composite methods for future research.

\subsection{Limitations}

As in any simulation study, a general limitation is that we consider only a small subset of all possible true models while the models generating empirical data may be much more complex. For example, the true models we considered did not include cross loadings and only included major factors. In some studies, numerous 'minor common factors' are added to the data-generating model that induce small covariances, so that there is a discrepancy between the population covariance matrix and the model's prediction in the population (i.e., model error; MacCallum, Widaman, Preacher, \& Hong, 2001; Tucker, Koopman, \& Linn, 1969). In this study, we considered sampling error but no model error, because it is somewhat arbitrary whether the minor factors should be considered 'error' or should be picked up by factor modeling techniques. So, while our simulation study involved a larger variety of true factor models compared to previous simulation studies, it would be interesting to expand the variety in true models further to investigate how the methods 
perform in cases where the data-generating model is more complex than the models we considered here.

The practice of including minor factors in the data-generating model and interpreting them as model error might explain differences between our results and the results of Preacher et al. (2013), who included such minor factors in their simulation set-up. For example, in Preacher et al.'s (2013) results, most selection techniques, such as the AIC, RMSEA and BIC for larger sample sizes, overfactored. This could be because these methods pick up some of the minor factors. If those minor factors are considered error and not part of the model, this results in overfactoring. In contrast, in our simulation study and the ones of Golino and Epskamp (2017), where no minor factors were included, all bias in selection techniques is due to underfactoring. This is also the case for the AIC, BIC and RMSEA that overfactored in Preacher et al.'s (2013) results (see Appendix $\mathrm{H}$, which presents the bias of all methods). A set-up with minor factors might favor techniques that are conservative in the number of factors it selects, while data generated without minor factors might favor techniques that are more liberal in the number of factors they select.

Another limitation of our simulation study is that we sampled continuous data, while data in psychological research are often polytomous. In Appendix E we demonstrate that our method performs well also when reducing the responses to $10,9, \ldots, 2$ categories. Both for the polytomous data and the continuous data we assume that the noise is drawn from an (underlying) multivariate Gaussian distribution, which implies that the distribution is unimodal and symmetric. If this assumption is not met, which would be the case for skewed variables, we would expect that the estimation of the correct number of factors would be more difficult. In this context, the results presented in this paper should be interpreted as a best-case scenario.

The premise of the simulation approach taken in this paper is that there is a true model that can in principle be estimated from data. We therefore only consider statistical methodology to determine the true number of factors. In practice, however, it may be appropriate to combine this statistical approach with domain knowledge. For example, factor solutions with certain numbers of factors might be implausible. Such knowledge can be used to restrict the range of considered numbers of factors (Preacher et al., 2013). However, domain knowledge could also be used after fitting a range of factor models. For example, one or several factors could be minor factors that are theoretically uninteresting. Here, one might choose based on substantive reasons to select a factor model with fewer factors, even though the minor factors are likely to provide a better fit in the population.

A promising direction for future work could be to use out-of-sample prediction based methods in other settings that require model selection between covariance matrices, such as in Structural Equation Modeling (SEM).

\subsection{Conclusions}

We have introduced a new method to estimate the number of factors in Exploratory Factor Analysis based on minimizing the out-of-sample prediction error of candidate factor models. We showed in an extensive simulation study that our new method outperforms existing methods including parallel analysis, model selection with the AIC, and Exploratory Graph Analysis by small margins. Critically, we also showed that our method has acceptable performance in all conditions, demonstrating robustness against the exact specification of the data generating factor model. Finally, we provide an implementation of our method in the R-package $f$ spe which is available on the Comprehensive R Archive Network (CRAN).

\section{Acknowledgements}

We would like to thank Fabian Dablander, Sacha Epskamp, Hudson Golino, Maarten Marsman, Mijke Rhemtulla and Lourens Waldorp for helpful comments on earlier versions of this paper. We would also like to thank three anonymous reviewers for their thoughtful and constructive comments that helped us to improve our paper. This work has been supported by the NWO Vici Grant no. 181.029 . 


\section{References}

Akaike, H. (1973). Maximum likelihood identification of gaussian autoregressive moving average models. Biometrika, 60(2), 255-265.

Akaike, H. (1998). Information theory and an extension of the maximum likelihood principle. In E. Parzen, K. Tanabe, \& G. Kitagawa (Eds.), Selected papers of Hirotugu Akaike (1st ed., pp. 199-213). Springer. https://doi.org/10.1007/978-1-4612-1694-0.

Bishop, C. M. (2006). Pattern recognition and machine learning. Springer. https://link .springer.com/book/9780387310732.

Browne, M. W., \& Cudeck, R. (1989). Single sample cross-validation indices for covariance structures. Multivariate Behavioral Research, 24(4), 445-455. https://doi.org/10.1207/ s15327906mbr2404_4.

Browne, M. W., \& Cudeck, R. (1992). Alternative ways of assessing model fit. Sociological Methods \& Research, 21(2), 230-258. https://doi.org/10.1177/0049124192021002005.

Campbell-Sills, L., \& Stein, M. B. (2007). Psychometric analysis and refinement of the ConnorDavidson resilience scale (CD-RISC): Validation of a 10-item measure of resilience. Journal of Traumatic Stress, 20(6), 1019-1028. https://doi.org/10.1002/jts.20271.

Carragher, N., Teesson, M., Sunderland, M., Newton, N., Krueger, R., Conrod, P., ... Slade, T. (2016). The structure of adolescent psychopathology: A symptom-level analysis. Psychological Medicine, 46(5), 981-994. https://doi.org/10.1017/S0033291715002470.

Cattell, R. B. (1966). The scree test for the number of factors. Multivariate Behavioral Research, 1(2), 245-276. https://doi.org/10.1207/s15327906mbr0102_10.

Crawford, A. V., Green, S. B., Levy, R., Lo, W.-J., Scott, L., Svetina, D., \& Thompson, M. S. (2010). Evaluation of parallel analysis methods for determining the number of factors. Educational and Psychological Measurement, 70(6), 885-901. https://doi.org/10.1177/ 0013164410379332.

Cudeck, R., \& Browne, M. W. (1983). Cross-validation of covariance structures. Multivariate Behavioral Research, 18(2), 147-167. https://doi.org/10.1207/s15327906mbr1802_2.

Diemer, M. A., Rapa, L. J., Park, C. J., \& Perry, J. C. (2017). Development and validation of the critical consciousness scale. Youth \& Society, 49(4), 461-483. https://psycnet.apa.org/ doi/10.1177/0044118X14538289.

Epskamp, S., Waldorp, L. J., Mõttus, R., \& Borsboom, D. (2018). The gaussian graphical model in cross-sectional and time-series data. Multivariate Behavioral Research, 53(4), 453-480. https://doi.org/10.1080/00273171.2018.1454823.

Fabrigar, L. R., Wegener, D. T., MacCallum, R. C., \& Strahan, E. J. (1999). Evaluating the use of exploratory factor analysis in psychological research. Psychological Methods, 4(3), 272-299. https://doi.org/10.1037/1082-989X.4.3.272.

Foygel, R., \& Drton, M. (2010). Extended Bayesian information criteria for Gaussian graphical models. In Advances in Neural Information Processing Systems 23 (NIPS 2010) (p. 2020-2028). Red Hook, NY: Curran. https://arxiv.org/abs/1011.6640.

Friedman, J., Hastie, T., \& Tibshirani, R. (2008). Sparse inverse covariance estimation with the graphical lasso. Biostatistics, 9(3), 432-441. https://doi.org/10.1093/biostatistics/ kxm045.

Glorfeld, L. W. (1995). An improvement on horn's parallel analysis methodology for selecting the correct number of factors to retain. Educational and Psychological Measurement, 55(3), 377-393. https://doi.org/10.1177/0013164495055003002.

Golino, H. F., \& Christensen, A. P. (2020). Eganet: Exploratory graph analysis - a framework for estimating the number of dimensions in multivariate data using network psychometrics [Computer software manual]. https://cran.r-project.org/web/packages/EGAnet/ EGAnet.pdf. (R package version 0.9.7)

Golino, H. F., \& Demetriou, A. (2017). Estimating the dimensionality of intelligence like data using exploratory graph analysis. Intelligence, 62, 54-70. https://doi.org/10.1016/j.intell .2017.02.007.

Golino, H. F., \& Epskamp, S. (2017). Exploratory graph analysis: A new approach for estimating the number of dimensions in psychological research. PloS one, 12(6), e0174035. https:// doi.org/10.1371/journal pone.0174035.

Golino, H. F., Shi, D., Christensen, A. P., Garrido, L. E., Nieto, M. D., Sadana, R., ... MartinezMolina, A. (2020). Investigating the performance of exploratory graph analysis and tradi- 
tional techniques to identify the number of latent factors: A simulation and tutorial. Psychological Methods, 25(3), 292.

Green, S. B., Levy, R., Thompson, M. S., Lu, M., \& Lo, W.-J. (2012). A proposed solution to the problem with using completely random data to assess the number of factors with parallel analysis. Educational and Psychological Measurement, 72(3), 357-374. https://doi.org/ $10.1177 / 0013164411422252$.

Guttman, L. (1954). Some necessary conditions for common-factor analysis. Psychometrika, 19(2), 149-161. https://doi.org/10.1007/bf02289162.

Harman, H. H. (1967). Modern factor analysis. University of Chicago Press.

Harshman, R., \& Reddon, J. (1983). Determining the number of factors by comparing real with random data: A serious flaw and some possible corrections. Proceedings of the Classification Society of North America at Philadelphia, 14, 14-15.

Haslbeck, J., \& van Bork, R. (2021). fspe: Estimating the number of factors in efa with out-ofsample prediction errors [Computer software manual]. https://cran.r-project.org/web/ packages/fspe/fspe.pdf. (R package version 0.1.0)

Hastie, T., Tibshirani, R., \& Friedman, J. (2001). The elements of statistical learning: Data mining, inference, and prediction (1st ed.). Springer. https://doi.org/10.1007/978-0 $-387-21606-5$.

Holzinger, K. J., \& Swineford, F. (1939). A study in factor analysis: The stability of a bi-factor solution. Supplementary Educational Monographs, no. 48.

Horn, J. L. (1965). A rationale and test for the number of factors in factor analysis. Psychometrika, 30(2), 179-185. https://doi.org/10.1007/BF02289447.

Kaiser, H. F. (1960). The application of electronic computers to factor analysis. Educational and Psychological Measurement, 20(1), 141-151. https://doi.org/10.1177/ 001316446002000116.

Kaiser, H. F. (1991). Coefficient alpha for a principal component and the Kaiser-Guttman rule. Psychological Reports, 68(3), 855-858. https://doi.org/10.2466/PR0.68.3.855-858.

Keith, T. Z., Caemmerer, J. M., \& Reynolds, M. R. (2016). Comparison of methods for factor extraction for cognitive test-like data: Which overfactor, which underfactor? Intelligence, 54,37-54. https://doi.org/10.1016/j.intell.2015.11.003.

Kohavi, R. (1995). A study of cross-validation and bootstrap for accuracy estimation and model selection. In International joint conference on artificial intelligence (pp. 1137-1145). http:// ai.stanford.edu/ ronnyk/accEst.pdf.

Lauritzen, S. L. (1996). Graphical models (Vol. 17). Clarendon Press. https://global.oup.com/ academic/product/graphical-models-9780198522195.

MacCallum, R. C., Widaman, K. F., Preacher, K. J., \& Hong, S. (2001). Sample size in factor analysis: The role of model error. Multivariate Behavioral Research, 36(4), 611-637. https://doi.org/10.1207/S15327906MBR3604_06.

Preacher, K. J., Zhang, G., Kim, C., \& Mels, G. (2013). Choosing the optimal number of factors in exploratory factor analysis: A model selection perspective. Multivariate Behavioral Research, 48(1), 28-56. https://doi.org/10.1080/00273171.2012.710386.

Revelle, W. (2021a). Package 'psychtools'. The comprehensive $R$ archive network. https:// cran.r-project.org/web/packages/psychTools/psychTools.pdf.

Revelle, W. (2021b). Package 'psych'. The comprehensive $R$ archive network. https://cran.r -project .org/web/packages/psych/psych.pdf.

Revelle, W., \& Rocklin, T. (1979). Very simple structure: An alternative procedure for estimating the optimal number of interpretable factors. Multivariate Behavioral Research, 14(4), 403414. https://doi.org/10.1207/s15327906mbr1404_2.

Rosseel, Y. (2012). Lavaan: An r package for structural equation modeling and more. Version 0.5-12 (BETA). Journal of Statistical Software, 48(2), 1-36. https://doi.org/10.18637/ jss.v048.i02.

Ruscio, J., \& Roche, B. (2012). Determining the number of factors to retain in an exploratory factor analysis using comparison data of known factorial structure. Psychological Assessment, 24(2), 282-292. https://doi.org/10.1037/a0025697.

Schwarz, G. (1978). Estimating the dimension of a model. The Annals of Statistics, 6(2), 461-464. http://dx.doi.org/10.1214/aos/1176344136.

Tucker, L. R., Koopman, R. F., \& Linn, R. L. (1969). Evaluation of factor analytic research procedures by means of simulated correlation matrices. Psychometrika, 34(4), 421-459. 
https://doi.org/10.1007/BF02290601.

Velicer, W. F. (1976). Determining the number of components from the matrix of partial correlations. Psychometrika, 41(3), 321-327. https://doi.org/10.1007/BF02293557.

Velicer, W. F., Eaton, C. A., \& Fava, J. L. (2000). Construct explication through factor or component analysis: A review and evaluation of alternative procedures for determining the number of factors or components. In R. Goffin \& E. Helmes (Eds.), Problems and solutions in human assessment (pp. 41-71). Springer, Boston, MA. https://doi.org/10.1007/ 978-1-4615-4397-8_3.

Watson, J. C. (2017). Establishing evidence for internal structure using exploratory factor analysis. Measurement and Evaluation in Counseling and Development, 50(4), 232-238. https:// doi.org/10.1080/07481756.2017.1336931.

Weng, L.-J., \& Cheng, C.-P. (2005). Parallel analysis with unidimensional binary data. Educational and Psychological Measurement, 65(5), 697-716. https://doi.org/10.1177/ 0013164404273941.

Zwick, W. R., \& Velicer, W. F. (1982). Factors influencing four rules for determining the number of components to retain. Multivariate Behavioral Research, 17(2), 253-269. https://doi.org/ $10.1207 / \mathrm{s} 15327906 \mathrm{mbr} 1702 \_5$. 


\section{A Estimating the Number of Factors with the fspe package}

Here we show how to use the implementation of our method in the R-package $f$ spe to estimate the number of factors in a data set containing ability measurements. Specifically, we use the classic psychometric data from Holzinger and Swineford (1939), which includes 24 measurements and has been taken from the R-package Psych Tools (Revelle, 2021a). Harman (1967) presents both a four and five-factor solution for this dataset. In the four-factor solution, the fifth factor corresponding to the variables 20-24 is eliminated. For this reason, we exclude variables 20-24, which gives us an example data set in which we would theoretically expect four factors.

We begin by loading the fspe package, which can be installed from the Comprehensive R Archive Network (CRAN), and the data, which are included in the package:

library (fspe)

data (holzinger19)

Next to providing the data to the fspe() function we specify that we we would like to consider models with up to 10 factors $(\operatorname{maxk}=10)$, that the cross-validation scheme should be performed 10 times $(\mathrm{rep}=10)$ with 10 folds $(\mathrm{nfold}=10)$, and that we would like to use the PE-method (method = "PE"). The alternative would be (method = "Cov"), for the CovE method. Finally, we set a seed so that the analysis shown here is fully reproducible.

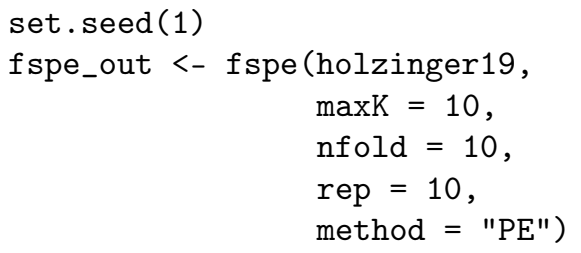

The estimated number of factors can be obtained from the output object:

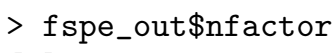

In addition, the output object contains the full prediction error path in $\mathrm{f}$ spe_out\$PEs, which we display in Figure 4:

\section{Figure 4}

The averaged (across variables and folds) prediction error for various candidate numbers of factors.

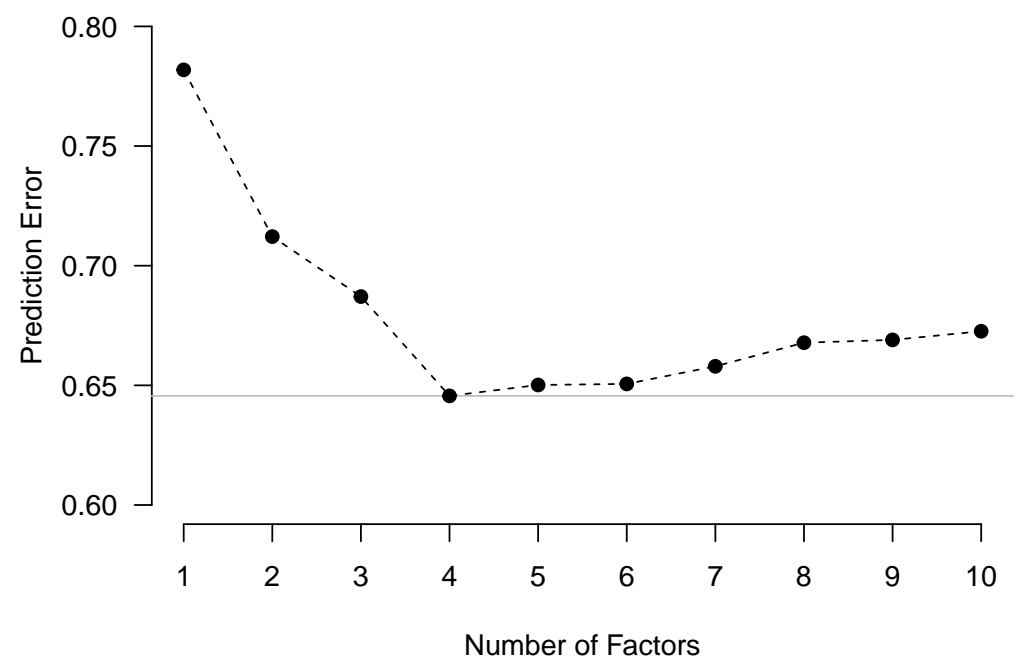

We see that the prediction error dramatically decreases from one factor through four factors, indicating that 1-3 factors are not enough to capture the factor structure in the data. However, when increasing the number of factors beyond four, the error increases again, since the additional flexibility of the model does not capture structure in the true model anymore, but is fitting noise instead. 


\section{B Performance of PE method as a Function of Folds}

In both the PE and the CovE method, we use cross-validation to approximate an out-of-sample error. A hyperparmeter in this cross-validation scheme is the number of folds. For both methods, we investigated the average accuracy of different choices of numbers of folds. To do so, we choose the cell with four true factors, three items per factor, $\Psi=0.4$ and $N=593$, because the overall performance in this cell is relatively low which renders it easier to detect a potential impact of fold size. We generated 1000 datasets from this cell and estimated the number of factors with our PE method using 2, 5, 10 and 20 folds. The average accuracy over the 1000 datasets as a function of folds is displayed in Figure 5:

Figure 5

Performance of PE and CovE methods as a function of the number of folds in cross validation.

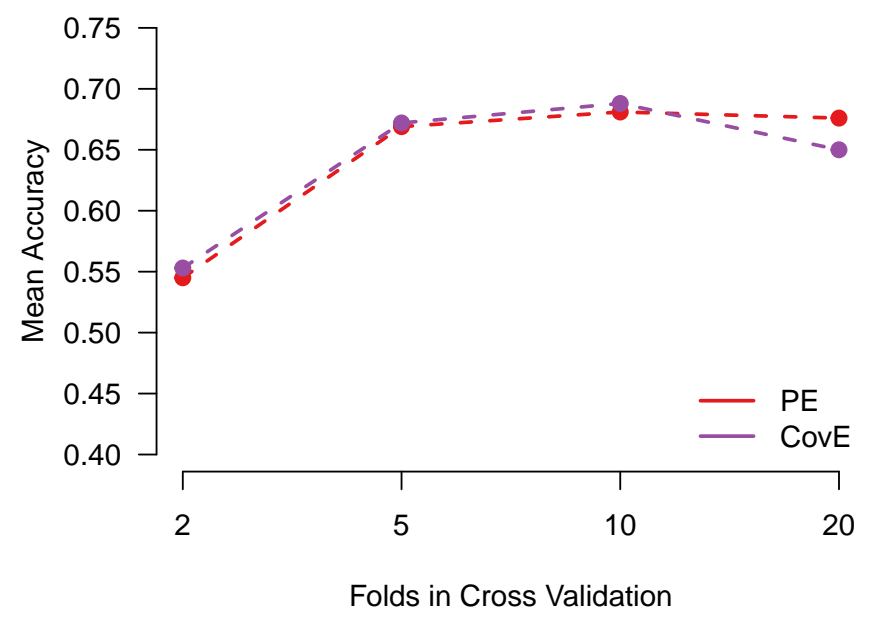

We see that the accuracy is highest for 10 folds both for PE and CovE. Consequently, we used 10 folds for both methods in the simulation reported in the main text.

\section{Performance of PE method as a Function of Repetitions}

A common strategy to improve the performance of model selection with cross-validation is to re-run the cross-validation scheme several times and aggregate the results to select a final model. This has the goal of averaging out the impact of randomness in the assignment of cases to folds. Here we use a majority rule to aggregate the estimated number of factors across repetitions. To investigate whether such repetitions increase the performance we study the same cell as in Appendix B with four true factors, three items per factor, $\Psi=0.4$ and $N=593$. Similarly to above, we choose this cell because we expect the maximum possible improvement due to repetitions in a cell where all methods tend to have moderate performance. In contrast, in cases in which all methods are doing extremely poorly or excellent, we would not expect to find a big impact of repetitions. Averaged across 100 iterations, we find that the version of PE with 10 repetitions has a $6.23 \%$ higher accuracy than PE with a single run. While we expect that this improvement will be on the upper end compared to other simulation cells, we think it warrants the inclusion of the 10-repetition version of $\mathrm{PE}$ (and CovE) in the main simulation.

\section{Performance of Parallel Analysis}

The results in Figure 2 show that parallel analysis performs poorly in conditions with small numbers of factors and variables per factor and small correlations between factors. This section provides a more detailed analysis of this perhaps surprising behavior.

In conditions in which parallel analysis performs poorly, the error is in most cases that the number of dimensions that is being selected is 0 . The reason is that the $95 \%$ quantile for the first 
eigenvalues that result from the simulated random data is much higher compared to their mean, than for other eigenvalues, which makes it more difficult for the real data to pass the threshold for the first eigenvalue. Figure 6 shows an example of the parallel analysis for a data set in the condition with 4 factors, 3 variables per factor, $\Psi=0$, and $n=1000$.

\section{Figure 6}

Example of parallel analysis with 4 factors, 3 vars/factor, sigma $=0$, and $n=1000$. In this example the test selects 0 factors because the first eigenvalue does not reach the threshold of the $95 \%$ quantile.

\section{Parallel test: 4 factors}

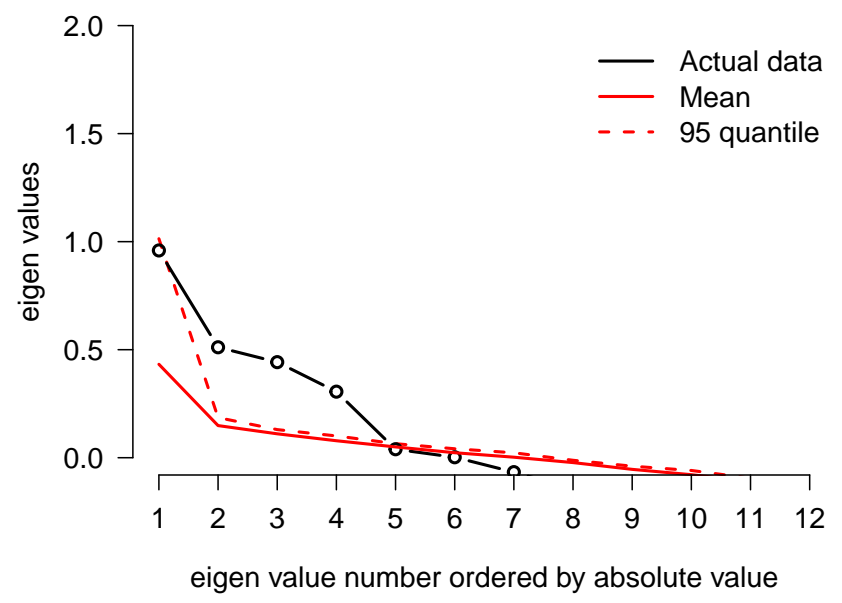

The red dotted line shows the $95 \%$ quantile of the eigenvalues of the simulated random data. The distance of this line to the mean of the eigenvalues of the random data (red solid line) is large for the first eigenvalue. In this example, the first eigenvalue of the real data is smaller than the $95 \%$ quantile, which resulted in 0 being selected as the number of dimensions, even though the 2nd, 3rd and 4th eigenvalue are larger than the 95\% quantile. So, if the first eigenvalue would have been slightly larger and reached the threshold, then 4 factors would have been selected.

The variance in the first eigenvalues of the random data can be decreased by increasing the sample size, however, huge sample sizes are required to do so. For example, Figure 7a shows a parallel analysis performed on a population correlation matrix that is consistent with 2 factors, 3 factors per variable, and correlations between the variables belonging to the same factor equal to 0.3 and correlations between variables of different factors equal to 0 . The data $(n=1000)$ are generated without sampling error, and yet the parallel test selects 0 dimensions, instead of 2 . Even though the actual data do not contain sampling error, increasing the sample size still influences the parallel analysis through the reference distribution based on the random data (with an infinite sample size the reference distribution is a flat line at zero). In this example, increasing the sample size to $n=50000$ does decrease the mean, but the $95 \%$ quantile is still equally high, resulting in 0 dimensions being selected (Figure 7b). Increasing the sample size even further at some point pulls the $95 \%$ quantile down, so that the parallel analysis correctly identifies 2 dimensions. For example, with $n=70000$, parallel analysis gives mixed results, and with $n=100000$ it mostly correctly picks 2 dimensions (Figure 7c).

While improving the performance through larger sample sizes requires enormous sample sizes, the performance improves quickly for conditions with stronger correlations (or factor loadings) because those push the first eigenvalue of the actual data above the threshold. Figure 8 shows the results of performing the parallel analysis to three datasets of $n=1000$, all three generated from the same population correlation matrix as used for Figure 7, except that the correlations of 0.3 are increased to 0.4 in Figure 8b, and to 0.5 in Figure 8c. 


\section{Figure 7}

Example of parallel analysis performed on data with different sample sizes generated without sampling error from the same population correlation matrix. The population matrix corresponds to a factor model with two orthogonal factors, and three variables per factor. The correlations between variables belonging to the same factor all equal exactly 0.3. The sample size does not influence the eigenvalues of the actual data (black line) since these were generated without sampling error, but does influence the reference distribution (red lines).

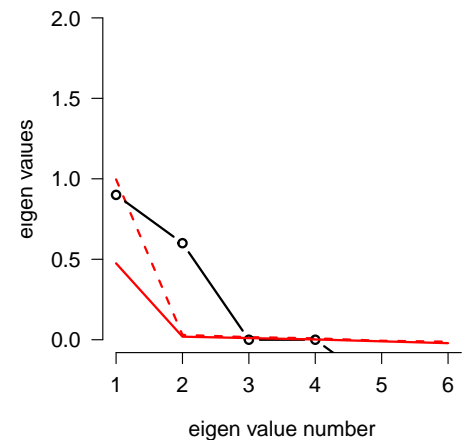

(a) $n=10,000$. Parallel analysis selects 0 factors.

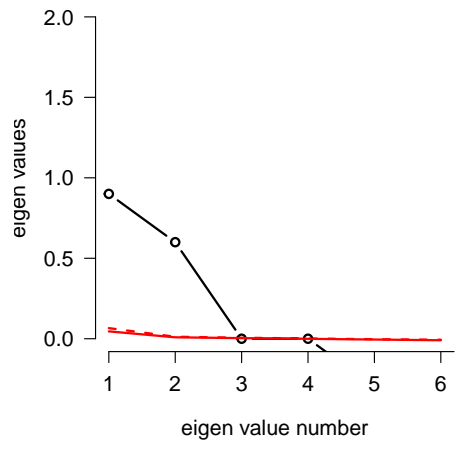

(b) $n=50,000$. Parallel analysis selects 0 factors.

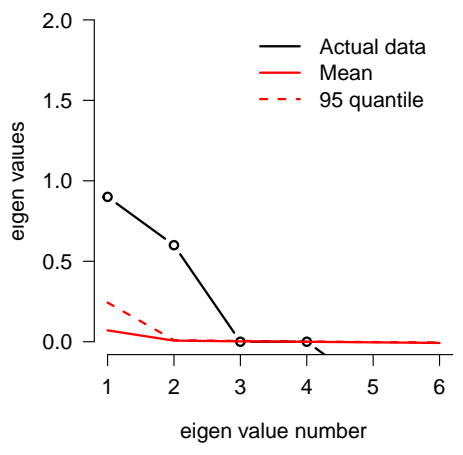

(c) $n=100,000$. Parallel analysis selects 2 factors.

\section{Figure 8}

Example of parallel analysis performed on data $(n=1000)$ generated without sampling error from a population correlation matrix that corresponds to a factor model with two orthogonal factors, and three variables per factor. The correlations between variables belonging to the same factor all equal exactly $0.3(8 a), 0.4(8 b)$ or $0.5(8 c)$. The increased correlations influence the eigenvalues of the actual data (black line) and do not influence the reference distribution (red lines).

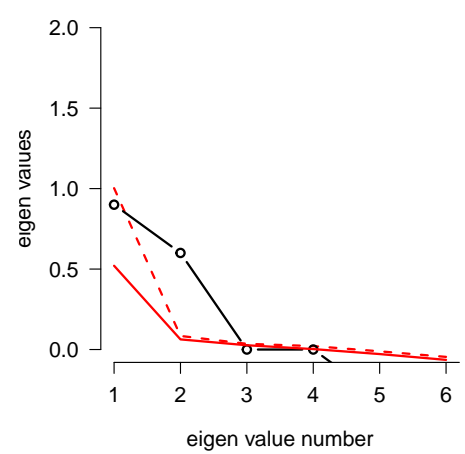

(a) $r=0.3$. Parallel analysis selects 0 factors.

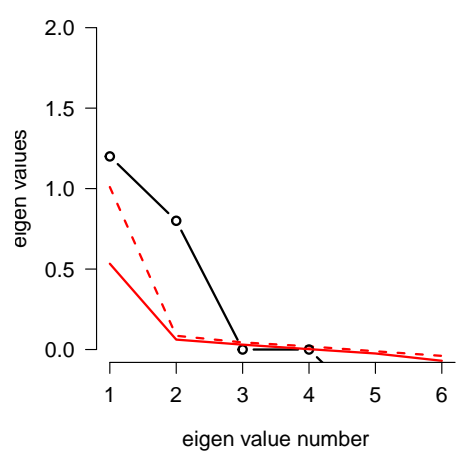

(b) $r=0.4$. Parallel analysis selects 2 factors.

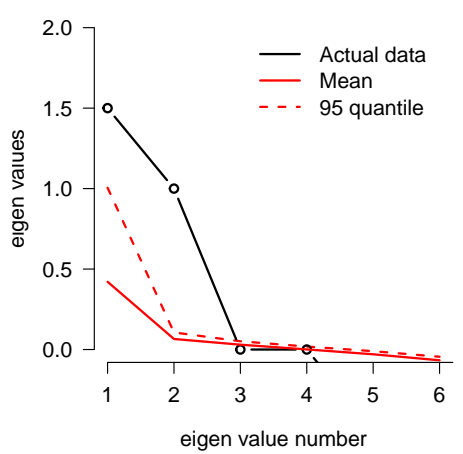

(c) $r=0.5$. Parallel analysis selects 2 factors.

Thus, the poor performance of parallel analysis in the condition with $\Psi=0$, number of factors $=2$, and variables per factor $=3$, gets better when either moving to conditions with stronger correlations (e.g., higher factor loadings or higher values for $\Psi$ ) or moving to conditions with higher numbers of correlated variables (e.g., more correlated factors, or more variables per factor), because in these conditions the first eigenvalue of the actual data is large enough to meet the $95 \%$ threshold. Note that while increasing the correlation between factors boosts the performance of parallel analysis in these conditions with small numbers of variables and factors, it results in worse performance in other conditions where the first eigenvalue already meets the $95 \%$ threshold. In these conditions a stronger correlation between factors makes it more difficult to estimate the separate factors, which is similar to what we see for other methods. 


\section{E Performance for Polytomous Responses}

We evaluate whether our PE method is robust against having only a few response categories instead of continuous data. That is, how much does the performance of the PE method drop when assuming that the data are continuous, while they are actually polytomous? And how does this drop in performance compare to that of other methods?

To investigate this question we generate continuous data as in the simulation in the main text, but then collapse the responses into $2, \ldots, 10$ equally large categories. We then estimate the number of factors with these polytomous (or binary, for two categories) data using the three highest performing method, the PE method, parallel analysis, and EGA. We choose a true factor model with four factors, six variables per factor, a high correlation between factors of 0.8 , and $n=1000$ observations, since we have seen in Figure 2 that this condition provides a good contrast of the performance of all methods. Figure 9 shows the average accuracy over 200 iterations. We see that all methods show a significantly lower performance if there are only a small number of ordinal categories. For two and three categories, parallel analysis and EGA perform slightly better than PE. From four or five categories onwards, their performance is comparable.

\section{Figure 9}

The accuracy of PE, Parallel analysis and EGA as a function of the number of response categories, for a factor model with four factors, six variables per factor, a correlation between factors of 0.8 , and $n=1000$. "Cont." stands for a continuous response scale.

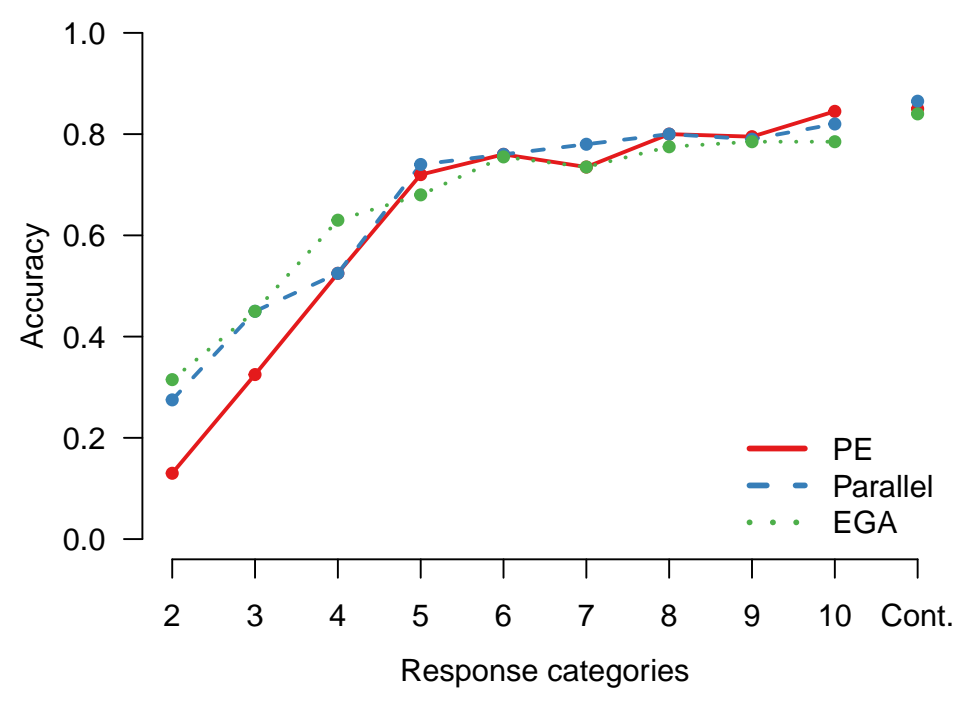

\section{F Summary of published simulation studies}

Table 1 provides a summary of the results and design of several simulation studies that have compared different techniques for identifying the number of factors. What method performs best differs across studies, but overall EGA and PA methods performed well in all studies. 


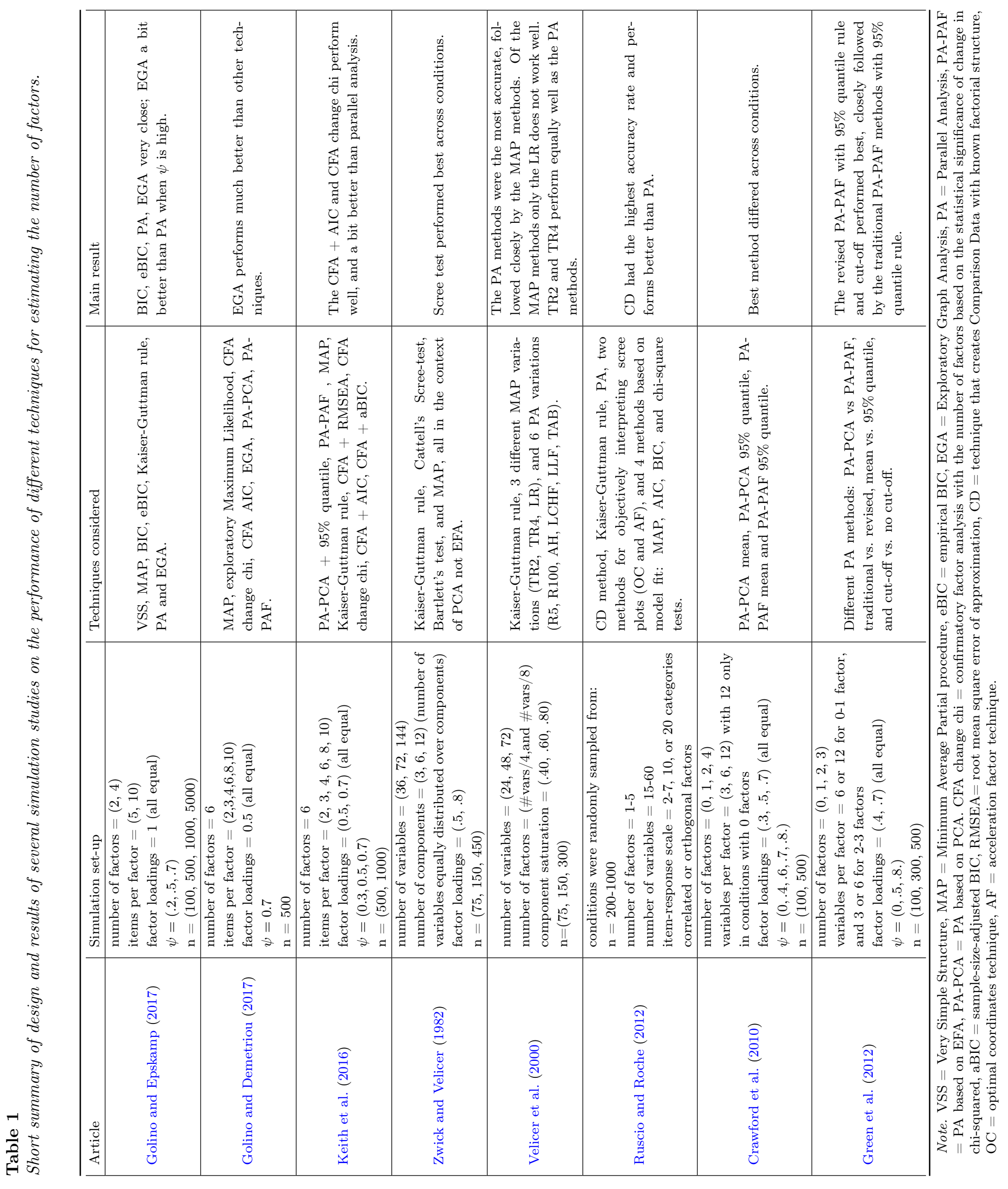




\section{G Performance of Composite Predictions}

Here we consider the performance of using a majority rule based on the Top $3, \ldots, 10$ performing methods shown in Figure 1 in the main text. In case of a draw, a prediction is randomly selected from the number of factors with the highest number of votes. We see that the prediction from the majority rule of the top $3,4,5,6$ and 7 methods outperforms our method by a small but significant margin.

\section{Figure 10}

The accuracy of a majority rule prediction of the Top 3, ..., 10 methods in Figure 1 in the main text. The dashed line indicates the performance of the PE method.

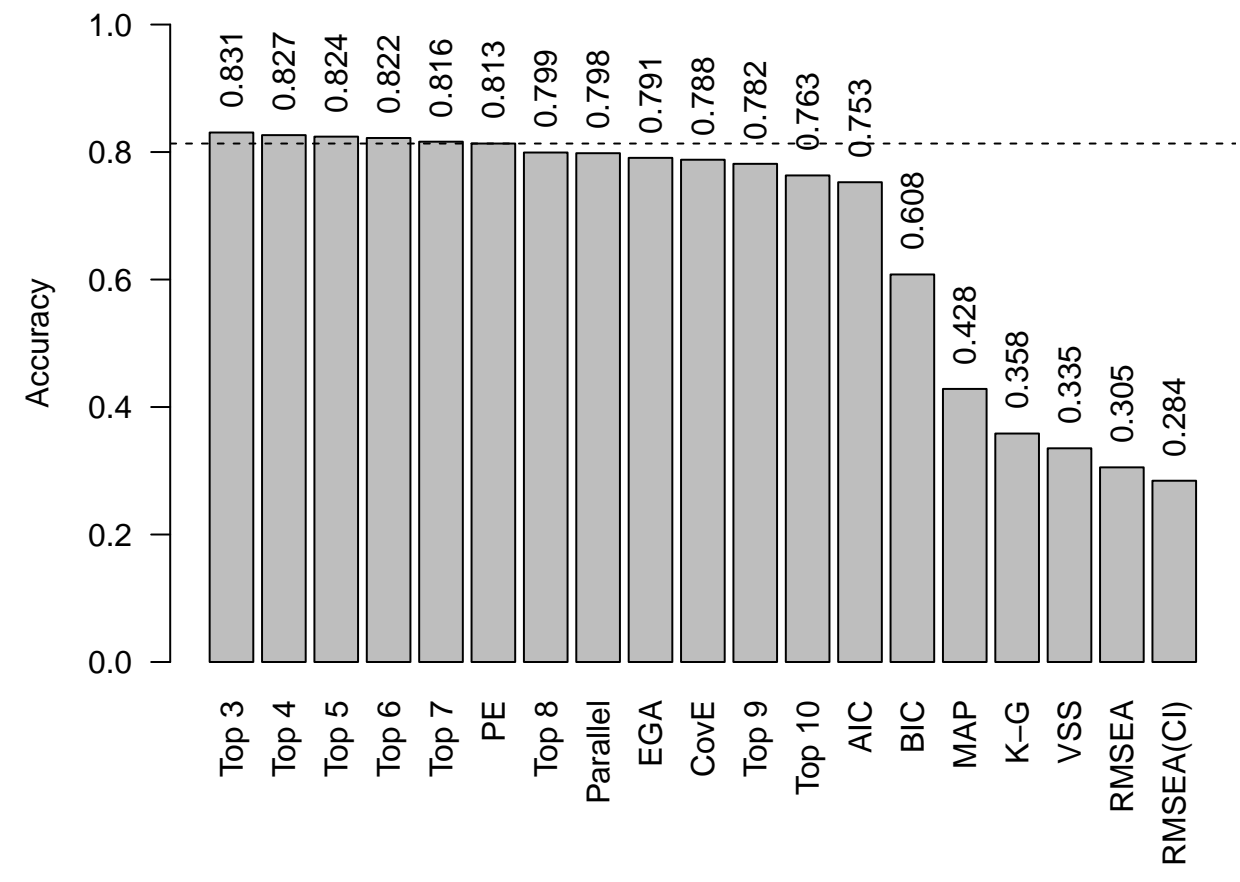

The composite predictions can outperform the best performing individual methods because among the best performing methods, the relatively poorly performing ones are in the minority across all scenarios. For example, the top 3 methods are PE, Parallel and EGA. With two factors and high correlations between factors, EGA performs extremely poorly, but PE and Parallel are performing well, leading to a high performance in the majority rule. Similarly, for two factors and low correlation between factors EGA and PE perform well and Parallel performs poorly, again leading to a high performance of the majority rule. 


\section{H Bias for Additional Estimation Methods}

Figure 11

The bias (true-estimated) of the five methods shown in Figure 3, in addition to the bias for RMSEA, RMSEA(CI) and the BIC.

Factors $=1$
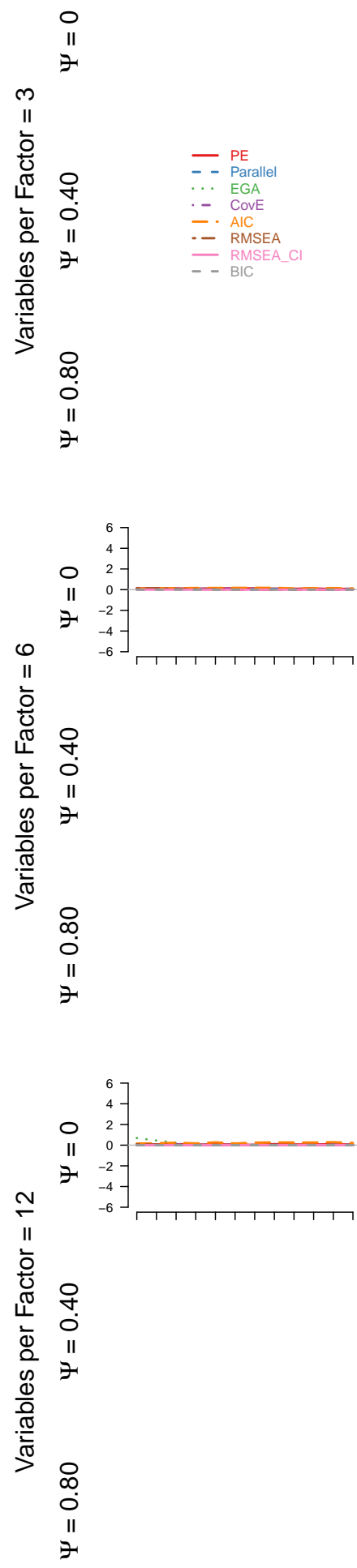

Factors $=2$
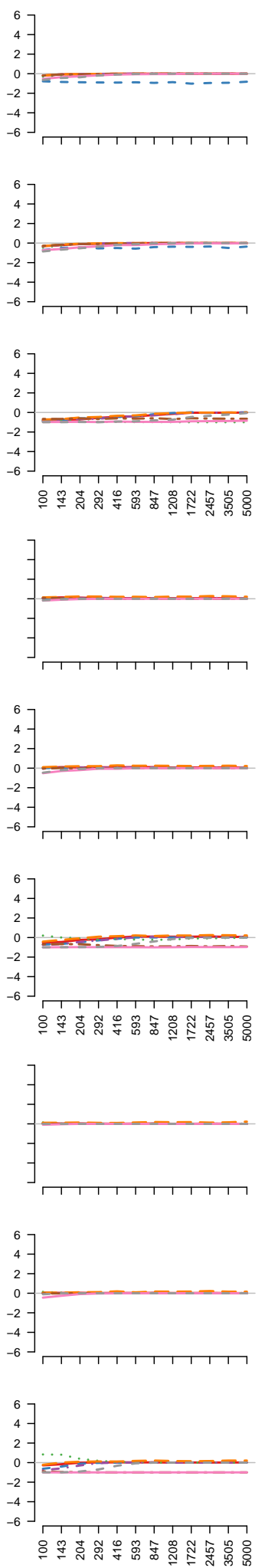

Factors $=4$
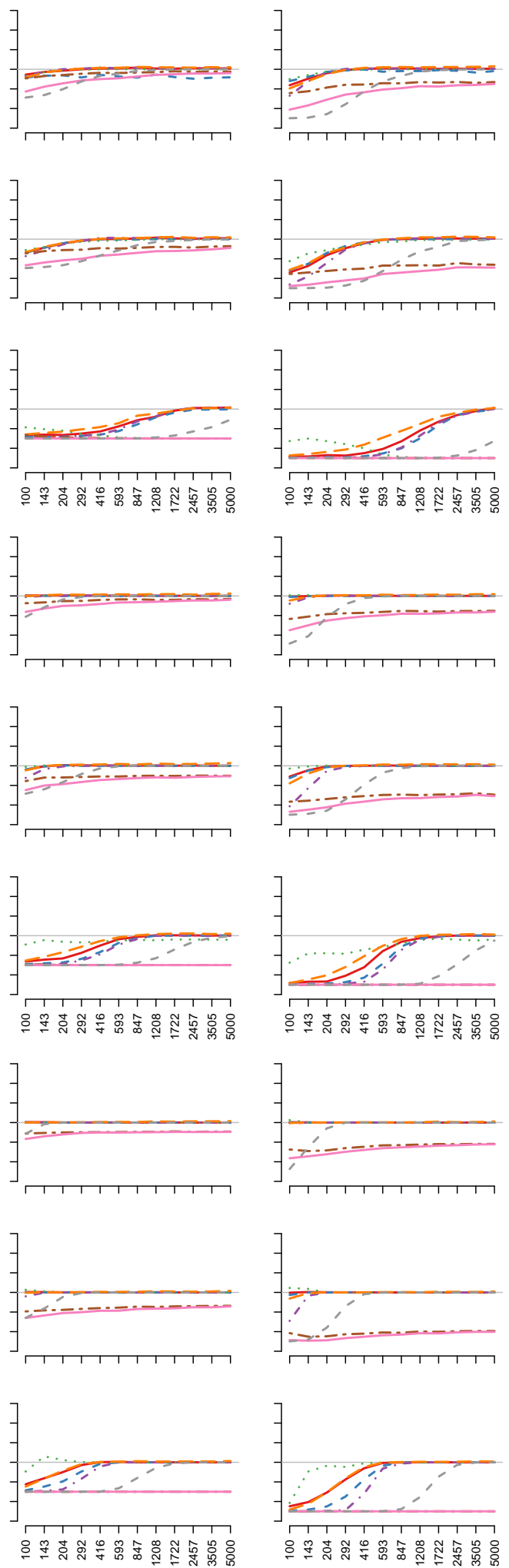